\title{
Visual-spatial and verbal-spatial binding in working memory
}

\author{
Jeanny Joana Rodrigues Alves de Santana ${ }^{1}$ and Cesar Galera ${ }^{2}$ \\ 1- Universidade Federal de Uberlândia, Uberlândia, MG, Brazil \\ 2- Universidade de São Paulo, Ribeirão Preto, SP, Brazil
}

\begin{abstract}
The asymmetric pattern of binding of features in working memory is a controversial topic in the literature. The binding of visualspatial and verbal-spatial information was studied in a serial recognition task to address the contribution of spatial location, visual appearance, and verbal identity to binding in working memory. The participants $(n=32)$ made a recognition judgment of two sequences of four stimuli each based on a relevant dimension while ignoring changes in an irrelevant dimension. In the visual and verbal tasks, the location was irrelevant. In the spatial tasks, the visual or verbal dimension was irrelevant. Our data showed that the visual or verbal characteristics of the object were incidentally encoded with the spatial location, but the spatial location of the items was not codified together with either the verbal features or visual characteristics when a verbal strategy was limited by articulatory suppression. This asymmetry in binding memory when the participants had to retain one of the features that was presented suggests a functional interaction between specific components of modalities of information and a system that maintains the multimodal representation. Keywords: working memory, binding, visual-spatial, verbal-spatial.
\end{abstract}

Received 04 September 2013; received in revised form 28 October 2014; accepted 30 October 2014. Available online 25 November 2014.

\section{Introduction}

Visuospatial working memory is widely accepted to encompass two storage systems. The visual store is responsible for storing and processing information related to shape, color, brightness, and static visual layout properties. The spatial system is related to storage locations and trajectories (Logie, 1995; Klauer \& Zhao, 2004; Darling, Della Sala, \& Logie, 2007). Once established, the functional dissociation between the visual and spatial storage systems presents a new problem. At some point, visual and spatial information must be integrated so we can remember, for example, the location of an object, or if it was a green square or a red triangle. Experimental evidence shows that the ability to store integrated objects appears to require no more attention than the storage of its individual features alone, suggesting that the integration (i.e., binding) of color and shape occurs automatically (Allen, Baddeley, \& Hitch, 2006; Allen, Hitch, \& Baddeley, 2009; Karlsen, Allen, Baddeley, \& Hitch, 2010). In addition to being automatic, on some occasions the conjunction

Jeanny Joana Rodrigues Alves de Santana, Institute of Psychology, Universidade Federal de Uberlândia. Cesar Galera, Department of Psychology, Faculdade de Filosofia, Ciências e Letras de Ribeirão Preto, Universidade de São Paulo. Correspondence regarding this article should be directed to: Cesar Galera, University of São Paulo, Faculty of Philosophy, Sciences and Letters of Ribeirão Preto, Department of Psychology, 3900 Bandeirantes Avenue, Ribeirão Preto, SP, 14040-901, Brazil. Phone: +55-16-3602-3760, +55-16-36024393. Fax:+55-16-3602-3669. Email: algalera@usp.br appears to happen incidentally (i.e., the conjunction happens even when it is irrelevant to the task). For example, Jiang, Olson, and Chun (2000) and Olson and Marchuetz (2005) showed that the recognition of a visual stimulus was impaired when the position of a test stimulus was different from the position where the stimulus was stored. Thus, spatial information was encoded with visual information incidentally, although the participants were instructed to ignore the position where the visual stimuli were presented.

Another issue concerning the binding process is the nature of the representation that is stored in working memory. The dispute here is among representational and associative binding approaches. According to the representational perspective, there are two binding mechanisms: one involved in the coding of characteristics and the other involved in maintaining the integrated object (Ueno, Mate, Allen, Hitch, \& Baddeley, 2011). One assumption was that a filter based on characteristics is required to eliminate the distractors. Once an irrelevant characteristic gains access to the visual-spatial system (i.e., after it passes through the filter), it is automatically recorded at the object level. The representation of this object then becomes vulnerable to loss because of the objects' overlapping processes.

According to the representational perspective, the integrated object is maintained in the episodic buffer. This buffer allows binding of the content of specific storage components and the content of longterm memory into multidimensional representations, yielding integrated objects that can be inspected and 
manipulated consciously (Baddeley, Allen, \& Hitch, 2010). According to this approach, the object is stored as a new code that is different from its constitutive characteristics. "It is assumed to be episodic in the sense that it is capable of holding episodes, integrated chunks of information that then became accessible to conscious awareness" (Baddeley et al., 2010, p. 229). The episodic buffer is considered a "purely passive system...that serves a crucial integrative role because of its capacity to bind information from a number of different dimensions into unitized episodes or chunks" (Baddeley, Allen, \& Hitch, 2011, p. 1399).

This episodic buffer approach can be designed in parallel with the proposition that "object files" are formed in memory, similar to what occurs during perception (Zimmer \& Ecker, 2010). These "object files" describe a temporary representation of the features that are associated with the objects that form an integrated object identity, becoming "object tokens." "The reinstatement of an object token is assumed to generate a feeling of familiarity" (i.e., "unspecific awareness that the object has been experienced before, but without any conscious access to details of that encounter" (Zimmer \& Ecker, 2010, p. 1067).

The framework of associative binding in memory is an alternative to the representational perspective. This approach suggests that binding is maintained by transient associative links between independently held features (Elsley \& Parmentier, 2009). The core argument of the associative approach concerns the asymmetric effect of binding in working memory. Based on the premise that the incidental conjunction of features occurs, when one feature is tested, the other one is expected to be automatically retrieved (Guérard, Morey, Lagacé, \& Tremblay, 2013). However, the literature shows that this is not true in all situations. Growing evidence indicates asymmetry in the conjunction of features in the simultaneous presentation of stimuli, such as in the studies by Maybery et al. (2009), Campo et al. (2010), and Morey (2009), and the sequential presentation of stimuli, such as in the studies by Guérard, Tremblay, and Saint-Aubin (2009), Morey and Mall (2012), and Guérard et al. (2013).

Although some mechanisms of memory of simultaneously presented stimuli are known to be similar to those in which items are presented sequentially (Allen, Baddeley, \& Hitch, 2014), unknown is whether sequential presentation interferes with the maintenance of the bound representation. Previous evidence indicated that serial order is encoded incidentally with spatial, visual, and bound information (Santana \& Galera, 2013). Moreover, evidence suggests that the serial position of items is a factor that affects the integration of features (Guérard et al., 2013). The type of relationship that is established between features for the representation of the integrated object can be questioned. In the sequential presentation of items, a certain feature may be advantageous for memory when the irrelevant dimension changes between the sequence that is presented for memorization and the sequence that is presented for testing. What would this pattern tell us about the mechanism of binding in memory?

Considering the evidence on the asymmetrical contribution of features to binding in the simultaneous presentation of stimuli (Maybery et al., 2009; Morey, 2009; Campo et al., 2010), the present study investigated the following: (a) When stimuli are presented one by one, are the features that compose the objects integrated incidentally? (b) If the incidental binding of features occurs, would there be a differential contribution of features to the integrated representation (i.e., would asymmetry in the conjunction also occur in the sequential presentation of events)?

We investigated these questions by studying the binding of visual-spatial and verbal-spatial information in a serial recognition task. The serial recognition task consists of the presentation of two sequences of items that are defined by a relevant dimension and separated by a retention interval. The participants were instructed to memorize items in the first sequence and state whether the items in the second sequence were the same or not the same as in the first sequence, basing their responses only on the relevant dimension. The visualspatial stimuli were consonants in different shapes (e.g., the same letter presented in different fonts) that were presented in random locations on the computer screen. The verbal-spatial stimuli were consonants that were arranged in random locations on the computer screen. The multimodal information was presented in a task that requires the memorization of only one dimension. Our hypothesis was that if changing the irrelevant dimension generally affects the memory of relevant information, then the two dimensions (relevant and irrelevant) are integrated into a multimodal code. If asymmetry in binding occurs (i.e., if only one of the dimensions is encoded incidentally), then this would corroborate the hypothesis that associative links are formed in the binding of information instead of a multimodal code (Treisman \& Zhang, 2006). We predicted that the features would be integrated incidentally (i.e., changing an irrelevant feature in the test sequence affects memory of the relevant feature). If the features contribute unequally to the formation of the integrated representation, then we predicted that the pattern of effects of changing the irrelevant feature depends on the relevant dimension type in the task. Asymmetry in the conjunction would be found if the memory for feature " $A$ " depends on feature "B," although the recall of feature "B" occurs independently of feature " $\mathrm{A}$ " (Guérard et al., 2013; Elsley \& Parmentier, 2009).

\section{EXPERIMENT 1}

\section{Methods}

\section{Participants}

The participants were 16 students from the University of São Paulo, Ribeirão Preto, SP, Brazil (eight women, 
eight men; age range, 21-31 years; $M=24.06$ years, $S D=3.58$ years). All of the participants had normal or corrected-to-normal vision. All of the participants provided informed consent prior to commencing the study, and all applicable ethical guidelines were followed.

\section{Apparatus and stimuli}

The verbal stimuli were eight capital letters (B, G, $\mathrm{M}, \mathrm{P}, \mathrm{S}, \mathrm{V}, \mathrm{X}$, and $\mathrm{Z}$ ) that were presented in the same graphical font $(40 \times 40$ pixels). The visual stimuli were letters that were presented in different graphical fonts in the same size as the verbal stimuli. Two hundred ninety-four visual stimuli were selected from a combination of 21 consonants and 14 graphical fonts. The spatial locations of the stimuli were determined from 16 possible spatial locations that were uniformly distributed in two concentric circles (the circles were hidden during presentation). The circles had radii of 13.5 and $9.2 \mathrm{~cm}$.

The fixation point (i.e., initiation warning signal) was a cross $(16 \times 16$ pixels $)$ that was located in the center of the screen. The auditory tone that signaled the presentation of the test was $1000 \mathrm{~Hz}$. The stimuli were presented on a 15 " monitor $(1024 \times 768$ pixel resolution $)$ in black $\left(0.92 \mathrm{~cd} / \mathrm{m}^{2}\right)$ on a white background $(70 \mathrm{~cd} /$ $\mathrm{m}^{2}$ ). The presentation of the stimuli and recording of responses were performed using E-Prime 1.2. software (Schneider, Eschman, \& Zuccolotto, 2002).

\section{Procedure}

The experiment had a 4 (visual-spatial, verbal-spatial, spatial-visual, spatial-verbal sequence) $\times 2$ (unchanged/ changed relevant dimension) $\times 2$ (unchanged/changed irrelevant dimension) repeated-measures factorial design. Memory was tested using a serial recognition procedure, in which the participants judged, based on a previously defined relevant dimension, whether a tested sequence was equal to a sequence that was presented previously. Each sequence was formed by four stimuli that were defined by the visual, verbal, and spatial information.

In trials in which the relevant dimension was visual or verbal, the irrelevant dimension was the spatial location of the stimuli. In trials in which the relevant dimension was the spatial location, the irrelevant dimension was visual or verbal. Thus, four types of stimuli were used and defined by combinations of relevant-irrelevant dimensions: visual-spatial, verbal-spatial, spatial-visual, and spatial-verbal. At the beginning of each session block, the participants were told about the relevant dimension of the memory task and instructed to explicitly ignore changes in the irrelevant dimension.

Each participant completed four blocks of trials. In the Visual Stimuli Block, the sequences were formed by the same letters but presented in different graphical fonts. In the Verbal Stimuli Block, the sequences were formed by different letters that were presented in the same graphical font. In these two blocks of trials, the participants were asked to judge whether the stimuli in the two sequences were the same, regardless of whether they were presented in the same spatial positions (irrelevant dimension). In both Spatial Blocks, the participants were asked to judge whether the stimuli of the two sequences occupied the same spatial positions, regardless of whether the stimuli of the two sequences were equal (irrelevant dimension). The stimuli were visual in one Spatial Block and verbal in the other. The relevant dimension changed in only half of the trials. In both cases, the irrelevant dimension was the same in only half of the trials (Figure 1). Whenever a change occurred, regardless of whether it was in the relevant dimension, the probability of presenting the first, second, third, or fourth stimulus was equal in all four serial positions. The order in which the blocks of trials were performed was counterbalanced among the participants.

In the sequence to be memorized and in the test sequence, each stimulus remained on the screen for $400 \mathrm{~ms}$, with an interstimulus interval of $100 \mathrm{~ms}$. The retention interval between sequences was $1000 \mathrm{~ms}$. After presentation of the second sequence, the participants responded by pressing the 1 key on the keyboard if the sequences were identical and the 2 key if the sequences were different. A feedback signal, lasting for 1000 $\mathrm{ms}$, was provided immediately after the participant's response. Correct responses were signaled by the word "Right," and incorrect responses were signaled by the word "Wrong."

Each participant performed 256 trials divided into four blocks of 64 trials each. In half of the trials (32 trials) the relevant dimension was identical in both sequences and different in the other half (32 trials). In the identical sequences, half of the trials (16 trials) presented the same irrelevant dimension, and half of the trials (16 trials) presented a different one. In half of the trials in which the sequences were different in the relevant dimension (16 trials), the irrelevant dimension did not change; in the other half, the irrelevant dimension changed in the test sequence. Forty practice trials were conducted, divided equally among four blocks.

\section{Results}

Accuracy $\left(A^{\prime}\right)$ in the four conditions, considering the unchanged/changed irrelevant dimension, and response bias in the experimental conditions ( $B$ ") were analyzed using analysis of variance (ANOVA).

The analysis of accuracy revealed significant effects of memory task $\left(F_{3,45}=63.68, p<.001, \eta_{p}^{2}=.80\right)$ and changes in the irrelevant dimension $\left(F_{1,15}=163.35\right.$, $\left.p<.001, \eta_{p}^{2}=.91\right)$ and an interaction between these two factors $\left(F_{3,45}=11.47, p<.001, \eta_{p}^{2}=.43\right.$; Figure 2). Performance was higher in verbal memory $(M=87 \%$, $S E M=2 \%)$ than in visual memory $(M=60 \%, S E M$ $=2 \%)$. In the spatial memory task, performance was similar, regardless of whether the irrelevant dimension was verbal or visual (both average accuracy $=72 \%$, 

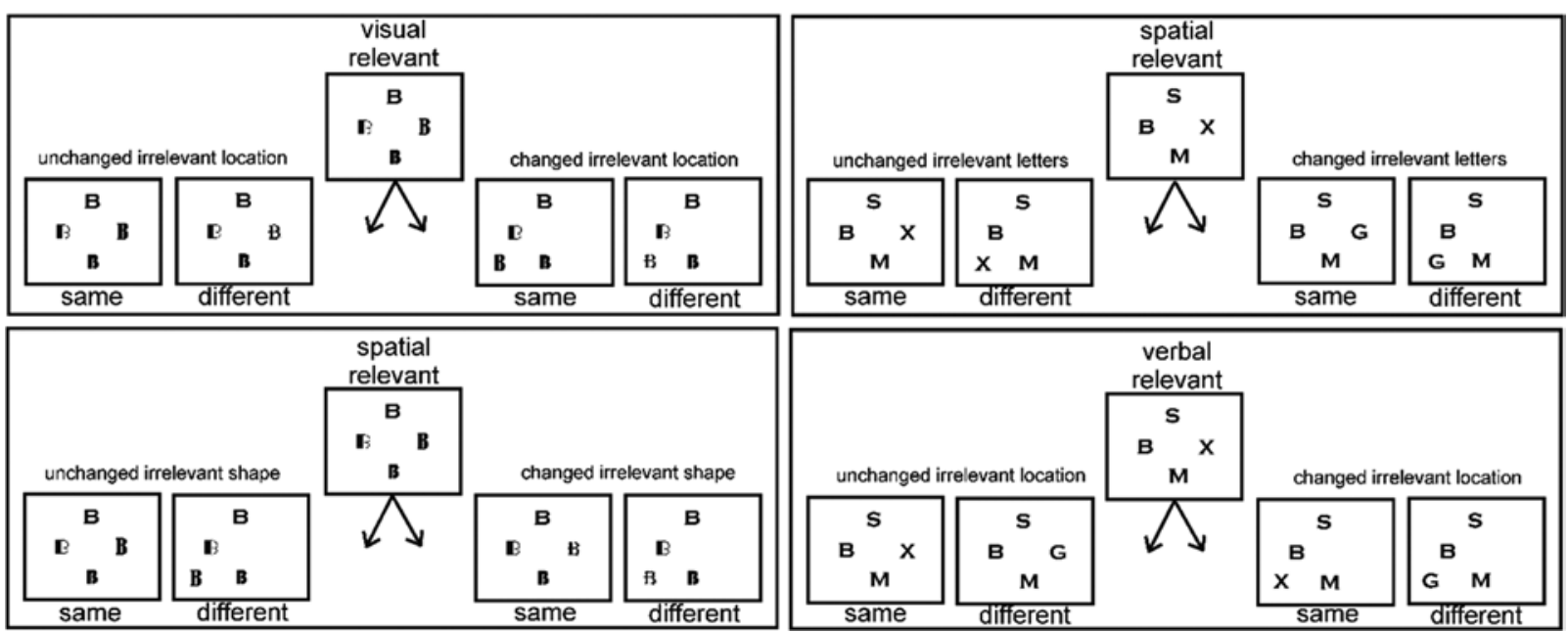

Figure 1. Example of trials from the four blocks of the memory tasks, represented by combinations of relevant and irrelevant dimensions. During the encoding and testing phases, the stimuli were presented sequentially but are shown here simultaneously to facilitate the representation of events in the experimental trials. The depiction is not to scale.

$\mathrm{SEM}=3 \%)$. Accuracy was higher when the irrelevant dimension changed $(M=78 \%, S E M=3 \%)$ than when it was the same $(M=67 \%, S E M=4 \%)$. The interaction showed that changing the irrelevant dimension increased performance in all of the memory tasks, with the exception of the verbal condition in which no improvement in accuracy was observed. A $t$-test showed no differences $(p>.05)$ in performance improvements by changing the irrelevant dimension in the visual-spatial task and spatial-visual task (interference magnitude).

The analysis of response bias (Figure 2) revealed a significant effect of changing the irrelevant dimension on recognition in the memory tasks $\left(F_{1,15}=21.75, p\right.$ $\left.<.001, \eta_{p}^{2}=.59\right)$ and an interaction between memory task and irrelevant dimension $\left(F_{3,45}=3.33, p=.03\right.$, $\left.\eta_{p}^{2}=.18\right)$. Generally, the negative value indicated a conservative criterion (Figure 2), mainly when the irrelevant dimension was the same between sequences $(M=-.26, S E M=.07)$ compared with when the irrelevant dimension changed $(M=-.02, S E M=.08)$. The interaction between memory task and irrelevant dimension was positive when the participants had to respond based on the spatial information and the irrelevant visual dimension changed $(M=.08)$ compared with the unchanged irrelevant dimension condition $(M=-.36)$. These data suggest that the participants tended to indicate that the test sequence and sequence to be memorized were the same, except when the visual irrelevant dimension changed in the spatial task in which the participants tended to indicate that the sequences were different. A $t$-test was used to make comparisons between the visual-spatial memory tasks when the relevant dimension was visual and when the relevant dimension was the spatial information. This analysis showed that switching the response bias when the irrelevant dimension changed was more evident in spatial-visual memory tasks than in visual-spatial tasks.

\section{Discussion}

The results of Experiment 1 showed that the irrelevant dimension was incidentally codified in memory with the relevant dimension. Higher performance was observed in the memory tasks when the irrelevant dimension changed
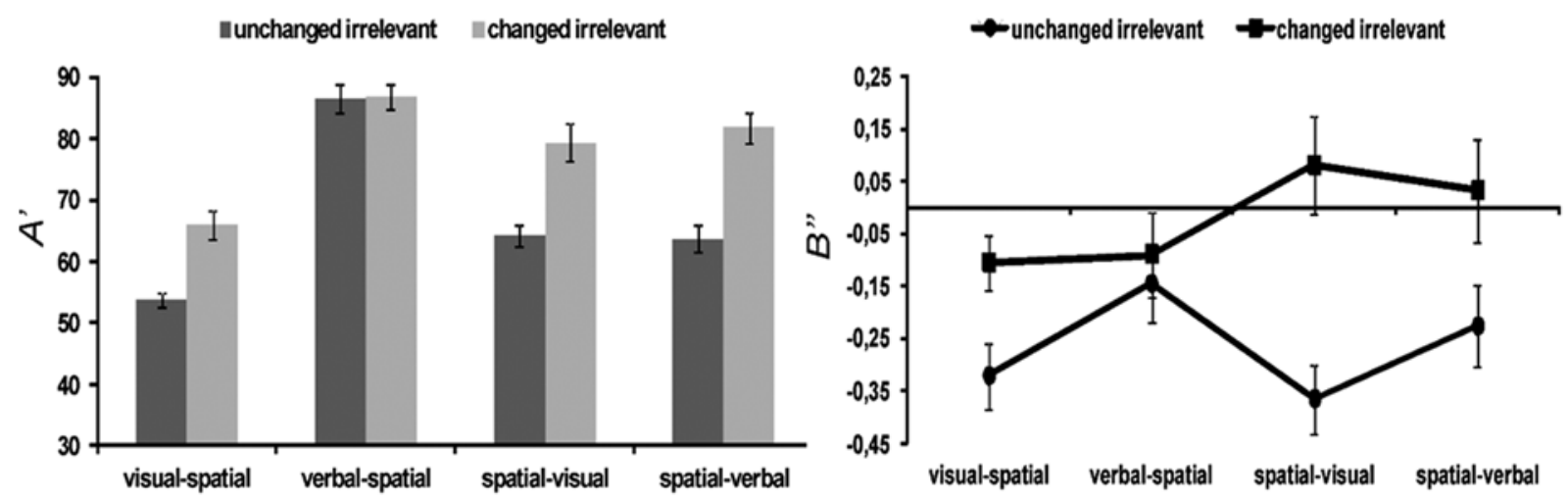

Figure 2. Accuracy $\left(A^{\prime}\right)$ and response bias $\left(B^{\prime \prime}\right)$ in Experiment 1 across memory tasks and the irrelevant dimension in the task. Bars represent the standard error of the mean. 
in the test sequence compared with when it did not change. One exception occurred with verbal memory in which performance did not vary throughout the irrelevant spatial conditions. These conclusions were based on the analysis of accuracy and response bias.

The participants had better performance in the conditions in which binding was broken, which appears to contrast with previous work (e.g., Treisman \& Zhang, 2006; Logie, Brockmole, \& Jaswal, 2011). Important details may explain the data. In these two previous studies, the tasks involved memory of the conjunction of two features and influence of location on the consolidation of this integration. Treisman and Zhang (2006), specifically experiments 1 and 2, evaluated the impact of manipulating the spatial location of isolated features of stimuli that were composed of shape-color and letter-color. Logie et al. (2011) studied the impact of changing the location of the shape-color conjunction. In the present study we evaluated shape-location and letter-location combinations. Furthermore, the pattern of results of these studies appears to be more complex than simply the observation that memory performance is better when the combination of features is preserved. As Treisman and Zhang (2006) reported, maintaining the same location of the stimuli between the presentations decreased memory performance when the conjunction of the features (shape-color, letter-color) was different between the presentation of the stimuli to be memorized and the test. To facilitate interpretation of the results, we presented the data in terms of performance accuracy $\left(A^{\prime}\right)$ rather than as a percentage of correct responses, as Treisman and Zhang (2006) had done.

We tested memory only for isolated features, but we also indirectly investigated whether another feature that was part of the object but was not relevant to the response is integrated incidentally with the irrelevant feature. Thus, we looked for indirect evidence of the conjunction of features, as Treisman and Zhang (2006) had done in experiments 1 and 2.

The effect of changing the irrelevant dimension was not the same throughout the tasks. Although the accuracy analysis indicated that the magnitude of the difference was equivalent between the changed and unchanged irrelevant dimension in the visual-spatial and spatial-visual conditions, the response bias analysis indicated different tendency criteria between these tasks. The magnitude of the effect of the irrelevant dimension, which was assessed by switching the response criteria when the irrelevant dimension changed, was more evident in the spatial-visual condition than in the visualspatial condition.

These data supported the prediction that visualspatial and spatial-verbal binding occurs without effort (Ueno et al., 2011). Nonetheless, verbal memory appears to be immune to the irrelevant spatial dimension. Additionally, the results suggested asymmetry in binding among the features in memory (Morey \& Mall, 2012; Guérard et al., 2013). The verbal information was incidentally codified with spatial information, but spatial information did not appear to be important for verbal memory, corroborating previous research (Maybery et al., 2009). The visual information was incidentally codified with spatial information, and spatial information was codified with visual information, but the response bias indicated different strategies in dealing with the irrelevant dimension. Working memory may have different types of binding, and the characteristics are bound in an associative way rather than in a representative manner (Elsley \& Parmentier, 2009).

The fact that the letters were recovered independently of spatial location may reflect a rehearsal mechanism that is present in the verbal condition that facilitated performance, despite changes in the spatial context. Because of this, it is necessary to determine whether the data on verbal memory are explained exclusively by a strategy of mentally rehearsing the letters that are presented visually. In Experiment 2 below, we clarified the role of verbal strategy in the codification of stimuli by introducing a demand for articulatory suppression during the encoding and maintenance of the sequence. We hypothesized that accuracy in the verbal task and visual task would be impaired if the shape of the letters was verbally rehearsed.

\section{EXPERIMENT 2}

\section{Methods}

\section{Participants}

Sixteen university students from the University of São Paulo, Ribeirão Preto, SP, Brazil, participated of this experiment (eight women, eight men; age range, 18 -30 years; $M=23$ years, $S D=3.38$ years). All of the participants had normal or corrected-to-normal vision. All of the participants provided informed consent prior to commencing the study, and none had participated in the previous experiment.

\section{Apparatus, stimuli, and procedure}

The apparatus, stimuli, and procedure were identical to those in Experiment 1, with the exception that before presenting the fixation point at the beginning of the trial, a sequence of digits (e.g., "1, 2, 3, 4, 5") was presented that the participants had to repeat aloud until the end of the maintenance interval. Afterward, an auditory tone signaled presentation of the test sequence.

\section{Results}

As in Experiment 1, accuracy and response bias were analyzed. The analysis of accuracy (Figure 3 ) revealed main effects of memory task $\left(F_{3,45}=15.20, p<.001, \eta_{p}^{2}\right.$ $=.50)$ and changes in the irrelevant dimension $\left(F_{1,15} \stackrel{p}{=}\right.$ 48.84, $\left.p<.001, \eta_{p}^{2}=.76\right)$ and a significant interaction between these factors $\left(F_{3,45}=32.92, p<.001, \eta_{p}^{2}=.68\right)$, replicating the results of Experiment 1. Performance was the worst in the visual memory task $(M=55 \%, S E M$ $=1 \%$ ) compared with the other tasks, and changing the irrelevant dimension caused better performance 

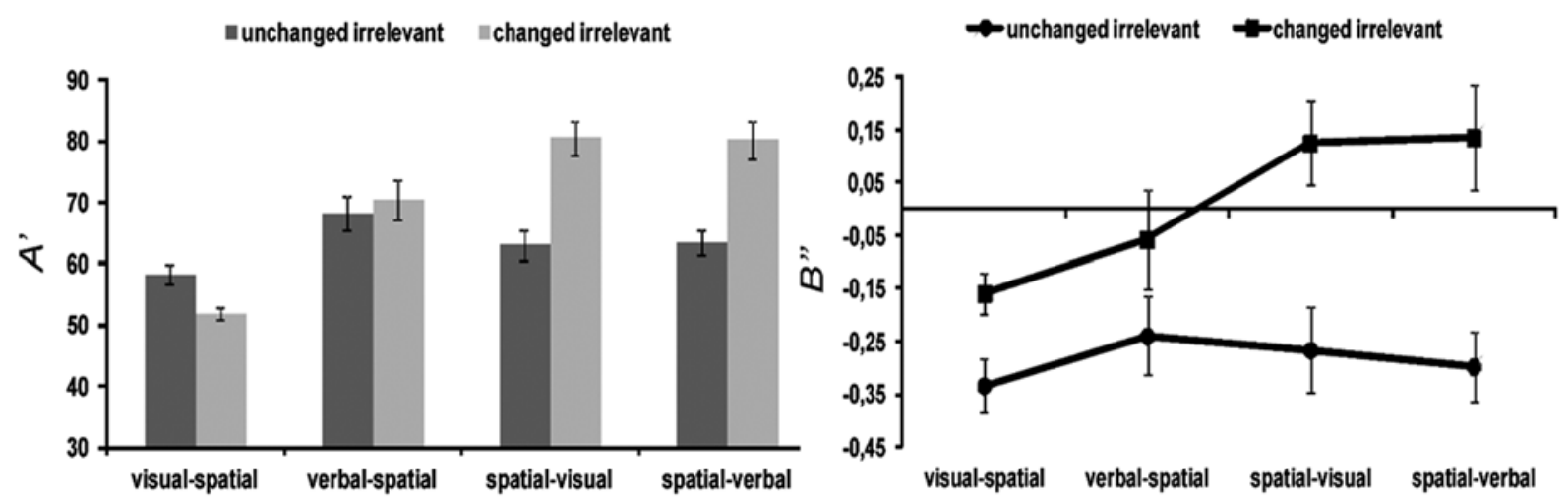

Figure 3. Accuracy $\left(A^{\prime}\right)$ and response bias $\left(B^{\prime \prime}\right)$ in Experiment 2 across memory tasks and the irrelevant dimension in the task. Bars represent the standard error of the mean.

$(M=70 \%, S E M=4 \%)$ compared with the unchanged irrelevant dimension condition $(M=63 \%, S E M=2 \%)$. The memory task $\times$ irrelevant dimension interaction showed that changing the irrelevant dimension improved spatial memory performance in general in both the visual and verbal irrelevant dimensions compared with the unchanged irrelevant dimension but did not have an effect in the verbal and visual memory tasks.

The analysis of response bias (Figure 3 ) revealed main effects of memory task $\left(F_{3,45}=2.91, p=.04, \eta_{p}^{2}\right.$ $=.16)$ and changes in the irrelevant dimension $\left(F_{1,15}=\right.$ 28.59, $\left.p<.001, \eta_{p}^{2}=.66\right)$ but no interaction between factors. The participants had a conservative criterion to respond in the visual memory task $(M=-.26, S E M=$ $.05)$ than in the spatial-visual $(M=-.08, S E M=.06)$ and spatial-verbal $(M=-.07, S E M=.07)$ tasks. This conservative criterion was generally observed in the condition when the irrelevant dimension was unchanged in the test sequence $(M=-.28, S E M=.05)$ compared with when it changed, the criterion of which tended to be liberal $(M=.01, S E M=.05)$.

\section{Discussion}

The data from Experiment 2 showed that articulatory suppression impaired the strategy of verbally rehearsing the visual and verbal stimuli. This was reflected by performance accuracy. The different criteria that were used among the memory tasks were reflected by response bias. Based on the accuracy analysis, we conclude that incidental binding occurred between the spatialverbal and spatial-visual conditions but not between the visual-spatial and verbal-spatial conditions. Response bias differed between memory tasks, reflected by a conservative criterion in the visual condition compared with the two types of spatial tasks. Similar to Experiment 1 , incidental binding occurred in working memory, but this binding depended on the relevant dimension in the task, presenting an evident asymmetric pattern.

The worst visual memory performance occurred when the change in spatial information was opposite to the previous experiment. The occurrence of some kind of verbal recoding of the irrelevant spatial information is assumed, in which articulatory suppression functions as an additional demand on memory, thus impairing performance. Morey et al. (2013) argued that once the binding occurs, this representation becomes more vulnerable to cross-interference than the memory of isolated features. Otherwise, binding with the rehearsal feature is assumed to be provided by a mechanism that utilizes resources that are external to those that are involved with the features that are being integrated. To draw more precise conclusions, assessing interference from intrinsic and extrinsic dimensions may be necessary.

\section{Experiment 1 and 2: analysis of unchanged relevant dimension}

The data from Experiments 1 and 2 were analyzed by considering only the trials in which the relevant dimension was unchanged in the sequence test and changes in the irrelevant dimensions. The analysis of correct responses revealed an interaction between the type of task and irrelevant dimension $\left(F_{3,90}=3.01\right.$, $\left.p=.034, \eta_{p}^{2}=.09\right)$. Changing the spatial information detrimentally affected visual memory performance $(M=$ $75 \%$ ) compared with maintaining the spatial information $(M=84 \%)$. This effect was not verified with the verbal or spatial relevant dimension.

This result is consistent with Treisman \& Zhang (2006) and speaks to the issue of binding asymmetry. Spatial information was important for visual memory, but spatial information was retained without reference to visual information. This result should be justified by the salience of the information or by the mechanism of functional interactions between components of working memory that are mediated by attentional resources.

\section{General discussion}

In the present study, we investigated the binding of visual-spatial and verbal-spatial information in working memory. A serial recognition task was used, in which the participants judged whether two sequences of stimuli were the same or different according to a relevant dimension. Our results showed that the identity 
of the objects in a sequence, either visual or verbal, was incidentally encoded with their spatial location, but the spatial location was not integrated with the verbal information. The spatial location was integrated with the visual information, but spatial interference in visual memory depended on the verbal strategies that were used to encode the information.

The differences observed in the binding patterns of visual and spatial information suggest that this type of binding was asymmetric. The binding was weaker in the visual relevant dimension compared with the spatial relevant dimension. When the visual task became more difficult (limited by a verbal strategy), changing the spatial information affected performance. Articulatory suppression appeared to impose an addition demand on working memory, which can affect performance not because of the verbal nature of the interference itself but rather because of disruption of the distribution of resources in the tasks. These results indicate a weakness of the implicit memory conjunction, indicating that the integrated nature of the representation is different from the maintenance of isolated features, as demonstrated in a previous study (Baddeley et al., 2011). To investigate this issue further, we need to evaluate the effects of different types of interference on visual and spatial memory (e.g., visual similarity and spatial configuration).

Additionally, the differences observed in the binding of verbal and spatial information suggest another asymmetry in working memory. The verbal system is immune to changes in the spatial location of stimuli in the case of verbal-spatial binding. However, the opposite is not true. Changing the verbal identity of the stimuli impaired the response for the spatial location of the items in the sequence. This effect was evident in Experiments 1 and 2. In the study by Campo et al. (2010), in contrast to our results, spatial and verbal binding occurred when the relevant dimension was verbal (i.e., changes in the location affected recognition of the letter, and changes in the letter did not affect memory of the location). These differences can be explained by the experimental conditions in the studies. In the study by Campo et al. (2010), the task required the recognition of just one item. A frame in the stimuli served as a mnemonic resource for the spatial information, and only the responses in the positive trials were analyzed. These differences may have become the spatial characteristic that predominated, without affecting binding. This means that both might be encoded. However, facilitation of the encoding or recitation of the spatial information may have occurred at the expense of the verbal information.

The verbal-spatial asymmetric binding that was observed in the present study suggests that verbal storage predominated over spatial storage. According to Morey (2009) and Morey and Mall (2012), these characteristics are stored separately but associated to preserve the binding, thus affecting responses in the task. If one of the systems stands out in terms of encoding strategies or recitation mechanisms, then the preponderance of one characteristic is observed, as demonstrated in the present study and in the study by Elsley and Parmentier (2009). To further investigate this issue, future studies should compare different types of task instructions that require attention to verbal and/or spatial features.

Morey and Mall (2012) explained asymmetry in verbal-spatial binding using a modified multi-component model of working memory. The episodic buffer would be responsible for maintaining the integrated object, but specific resources of the systems that are involved in binding would be associated with general domain resources to maintain the representation in conscious awareness. Morey and Mall (2012) suggested that the verbal storage component would benefit from a mechanism of information rehearsal that does not correspond to the spatial storage component. Considering our data in this context, the representation of verbal information would be "protected" by this rehearsal mechanism, thus preventing interference from irrelevant information. However, the spatial representation would not utilize this mechanism, thus causing greater vulnerability of the memory system to involuntary encoding of irrelevant verbal information. In this paradigm, storage systems are assumed to be specialized with regard to the modality of the stimulus, but an interaction between resources also occurs to maintain a multimodal representation. Thus, general domain resources that are involved in maintaining the integrated representation in the episodic buffer would be simultaneously used with specific features of the storage subcomponents.

The present results should be discussed in relation to methodological characteristics. One consideration is the necessity of determining whether the effects observed herein were attributable to interference with the encoding of stimuli or the response selection process and not the maintenance of information. Differences in the discrimination of stimuli may favor the encoding of one characteristic over another, and the spatial task could have been more difficult to perform than the verbal task (Maybery et al., 2009; Morey \& Mall, 2012). Consequently, the locus of interference would be placed on the simultaneous information encoding process rather than on maintenance of the content. Furthermore, access to the information and subsequent decisionmaking in the task occurred more accurately for one of the dimensions, thus generating different performance according to the combination of dimensions. These issues should be addressed in future studies.

In the present study, the data analysis was based on comparisons between positive trials that consisted of the replay of features and negative trials that included a new feature. This experimental method may have obscured the results because it may have influenced the response bias of the participants. The participants had a tendency to respond more accurately in trials in which a novel stimulus was presented (i.e., the detection of a new feature). This observation is reasonable but not entirely plausible because if the trial only evaluated the ability to detect a new feature, then the performance pattern would 
be identical in the four conditions in both experiments. However, we observed a discrepancy in performance between the verbal-relevant/spatial-irrelevant trials and spatial-relevant/verbal-irrelevant trials, in addition to a difference in visual memory performance between experimental conditions. To elucidate these issues, future studies should be designed to consider only positive trials, in which new features are not presented, and compare intact and recombined trials (Maybery et al., 2009).

These alternative explanations for the effects of interference between modalities were investigated by Cowan and Morey (2007). The participants in their study were exposed to sets of auditory stimuli or two sets of visual stimuli (colors). When the sets were presented in the verbal domain, they differed with regard to the voice that presented the stimuli (male or female) and nature of the stimuli (letters or digits). When the sets were presented in the visual domain, they differed with regard to shape (squares or circles) and location relative to the fixation point (oriented to the right or left of the fixation point). After the presentation of the two sets, an invalid cue appeared to indicate which set would be presented ("?" symbol), or a valid cue appeared to indicate whether the first or second set would be tested. Using this method, the authors differentiated the effects of encoding, maintenance, and response selection. The results showed that the detrimental effects of competing information on maintenance and not the encoding of this information were responsible for the impairment in memory performance in a relevant task. This conclusion was based on a specific experimental control in which the effect of maintaining the information was isolated from the conflict between encoding and response selection. We assume that the effects observed in the present study may be attributable to the maintenance of relevant and irrelevant information.

In summary, the present results demonstrate that visual-spatial and verbal-spatial binding can occur incidentally (i.e., without voluntary control of the integrated representation). An asymmetric relationship between the modalities in the storage of integrated characteristics was also observed. These data provide evidence that supports the multi-component working memory model in which there are specific systems for storing information. However, a functional interaction is assumed to occur between the components and the system (the episodic buffer) that maintains the multimodal representation, thus providing conscious awareness of the integrated object.

\section{Acknowledgements}

This research was supported by grants from Fundação de Amparo à Pesquisa do Estado de São Paulo (FAPESP; 2010/10913-5). The authors thank Ana Paula Uhlmann Corder and Mariana Sant'Anna Pereira for their assistance with data collection. We appreciate the comments of the reviewers and especially the advice of Prof. Dr. Ullrich Ecker.

\section{References}

Allen, R. J., Baddeley, A.D., \& Hitch, G. J. (2006). Is the binding of visual features in working memory resource-demanding? Journal of Experimental Psychology: General, 135(2), 298-313.

Allen, R. J., Baddeley, A. D., \& Hitch, G. J. (2014). Evidence for two attentional components in visual working memory. Journal of Experimental Psychology: Learning, Memory, and Cognition, 40(6), 1499-1509.

Allen, R. J., Hitch, G. J., \& Baddeley, A. D. (2009). Cross-modal binding and working memory. Visual Cognition, 17(1-2), 83-102.

Baddeley, A. D., Allen, R. J., \& Hitch, G. J. (2010). Investigating the episodic buffer. Psychologica Belgica, 50(3-4), 223-243.

Baddeley, A. D., Allen, R. J., \& Hitch, G. J. (2011). Binding in visual working memory: The role of the episodic buffer. Neuropsychologia, 49(6), 1393-1400.

Campo, P., Poch, C., Parmentier, F. B., Moratti, S., Elsley, J. V., Castellanos, N. P., ... Maestu, F. (2010). Oscillatory activity in prefrontal and posterior regions during implicit letter-location binding. Neurolmage, 49(3), 2807-2815.

Cowan, N. \& Morey, C. C. (2007). How can dual-task working memory retention limits be investigated? Psychological Science, 18(8), 686-688.

Darling, S., Della Sala, S., \& Logie, R. H. (2007). Behavioural evidence for separating components within visuo-spatial working memory. Cognitive Processing, 8, 175-181.

Elsley, J. V. \& Parmentier, F. B. (2009). Is verbal-spatial binding in working memory impaired by a concurrent memory load? Quarterly Journal of Experimental Psychology, 62(9), 1696-1705.

Guérard, K., Morey, C. C., Lagacé, S., \& Tremblay, S. (2013). Asymmetric binding in serial memory for verbal and spatial information. Memory and Cognition, 41, 378-391.

Guérard, K., Tremblay, S., \& Saint-Aubin, J. (2009). Similarity and binding in memory: bound to be detrimental. Quarterly Journal of Experimental Psychology, 62(1), 26-32.

Jiang, Y., Olson, I. R., \& Chun, M. M. (2000). Organization of visual short-term memory. Journal of Experimental Psychology: Learning, Memory, and Cognition, 26(3), 683-702.

Karlsen, P. J., Allen, R. J., Baddeley, A. D., \& Hitch, G. J. (2010). Binding across space and time in visual working memory. Memory and Cognition, 38(3), 292-303.

Klauer, K. C. \& Zhao, Z. (2004). Double dissociations in visual and spatial short-term memory. Journal of Experimental Psychology: General, 133(3), 355-381.

Logie, R. H. (1995). Visuo-spatial working memory. Hove, UK: Lawrence Erlbaum.

Logie, R. H., Brockmole, J. R., \& Jaswal, S. (2011). Feature binding in visual short-term memory is unaffected by task-irrelevant changes of location, shape, and color. Memory and Cognition, 39(1), 24-36.

Maybery, M. T., Clissa, P. J., Parmentier, F. B. R., Leung, D., Harsa, G., Fox, A. M., \& Jones, D. M. (2009). Binding of verbal and spatial features in auditory working memory. Journal of Memory and Language, 61, 112-133.

Morey, C. C. (2009). Integrated cross-domain object storage in working memory: Evidence from a verbal-spatial memory task. Quarterly Journal of Experimental Psychology, 62(11), 22352251.

Morey, C. C. \& Mall, J. T. (2012). Cross-domain interference costs during concurrent verbal and spatial serial memory tasks are asymmetric. Quarterly Journal of Experimental Psychology, 65(9), 1777-1797.

Olson, I. R., \& Marshuetz, C. (2005). Remembering "what" brings along "where" in visual working memory. Perception and Psychophysics, 67(2), 185-194.

Santana, J. J. R., \& Galera, C. (2013). Incidental encoding of the serial order of visual-spatial events in working memory. Spanish Journal of Psychology, 16, E65-E72.

Schneider, W., Eschman, A., \& Zuccolotto, A. (2002). E-Prime: reference guide. Pittsburgh: Psychology Software Tools.

Treisman, A., \& Zhang, W. (2006). Location and binding in visual working memory. Memory and Cognition, 34(8), 1704-1719.

Ueno, T., Mate, J., Allen, R. J., Hitch, G. J., \& Baddeley, A. D. (2011). What goes through the gate? Exploring interference with visual feature binding. Neuropsychologia, 49(23), 1597-1604.

Zimmer, H. D. \& Ecker, U. K. H. (2010). Remembering perceptual features unequally bound in object and episodic tokens: neural mechanisms and their electrophysiological correlates. Neuroscience and Biobehavioral Reviews, 34, 1066-1079. 